\title{
Subsurface Flows with Advancing Solar Cycle Using Dense-Pack Ring-Diagram Analyses
}

\author{
D. A. Haber, B. W. Hindman, J. Toomre
}

JILA and Dept. of Astrophysical and Planetary Sciences, University of Colorado, Boulder, CO 80309-0440, USA

R. S. Bogart

Solar Observations Group, HEPL-CSSA, Stanford University, Stanford, CA 94305-4085, USA

F. Hill

National Solar Observatory, Box 26732, Tucson, AZ 85726-6732, USA

\begin{abstract}
Ring-diagram analyses have become a powerful local helioseismic tool for studying large-scale flows in the upper solar convection zone. Using this technique on a dense-pack mosaic of many small regions extracted from the full-disk Doppler velocity data taken with the Michelson Doppler Imager (MDI) on SOHO, we study how the mean meridional and zonal flows vary with depth and latitude over the course of the advancing solar cycle from 1996 to 2000 .
\end{abstract}

\section{Ring-Diagram Analyses}

With the advent of high-resolution Doppler data from experiments such as MDI and the upcoming GONG+ upgrade, local helioseismic studies based on ringdiagram and time-distance analyses are providing insights into the dynamics of the upper convection zone. With ring-diagram analyses we typically track a small region of the sun as it rotates across the disk and deduce how the underlying average flow below that region varies with depth. The procedure involves a 3-D Fourier transform of the tracked region to generate a power spectrum. A cut at constant frequency in such a 3-D power spectrum of a sequence of Doppler velocity images reveals a nested set of rings, each corresponding to a different radial order of the acoustic wave field (see Figures $1 A$ and $B$ ). Displacements of the rings are produced by the advection of acoustic wave fronts by horizontal flows. In ring-diagram analyses we fit the power spectrum with a line profile that involves the two transverse ring displacements, the background power, and the mode's central frequency, width, and amplitude (Haber et al. 2000). We then use the measured ring displacements as input to an RLS inversion procedure to deduce the zonal and meridional flow components as a function of depth within the upper $14 \mathrm{Mm}$ of the convection zone. Each separate ring analysis assesses the average flow structure with depth below a $16^{\circ}$ region on the solar surface. Figure $1 C$ shows an example of how the horizontal flows vary with depth for a single inversion of a representative region at disk center.

We use full-disk Doppler velocity images that are available with nearly continuous coverage from the MDI Dynamics Programs. We employ a densepack strategy of ring diagrams to map the evolving horizontal velocities with 

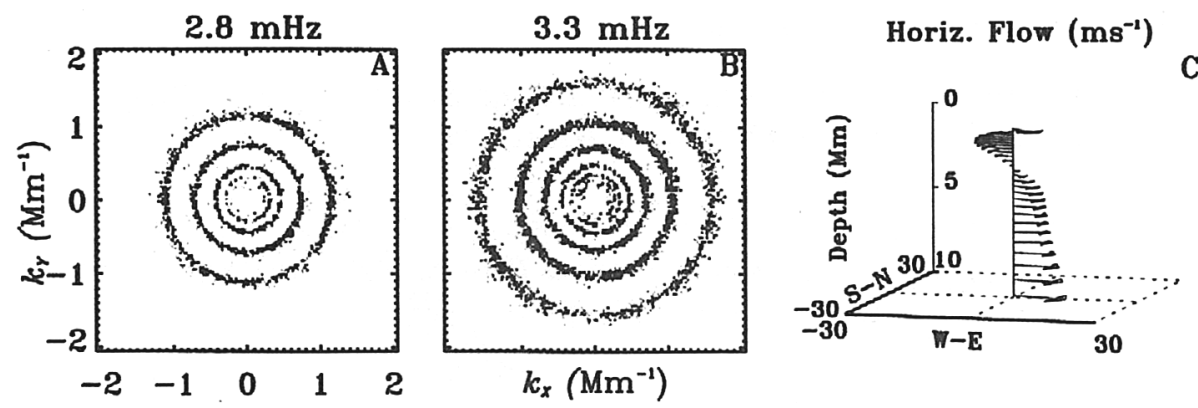

Figure 1. $\quad A, B$ Two cross-sectional cuts at constant frequency of a 3-D power spectrum showing variation of power with horizontal wavenumbers $k_{x}$ and $k_{y}$. The spectrum was generated by tracking a $16^{\circ}$ region for about 27.5 hours starting on 3 April 1999. $C$ Results of an inversion for the underlying horizontal flow with depth.

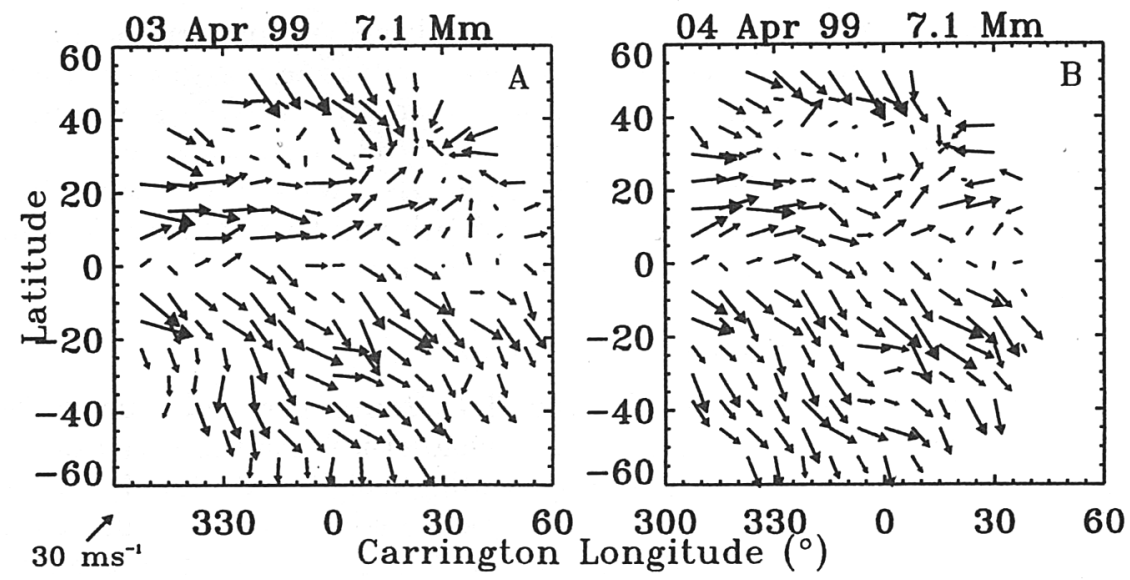

Figure 2. Dense-pack maps covering much of solar disk showing the horizontal velocities determined for a depth of $7.1 \mathrm{Mm}$ for two adjacent days of observation.

depth over a dense mosaic of overlapping tiles on the solar disk. The overall area analyzed extends about $53^{\circ}$ from disk center. The dense-pack tiling for each day consists of 189 separate regions tracked as they cross the disk for 1664 minutes $(27.5 \mathrm{hr})$. A full description of how such sets are generated is provided by Haber et al. (2000). Here we discuss the general findings of dense-pack studies and inversions carried out on a nearly daily schedule for at least two Carrington rotations ( $~ 50$ days) in 1996 and 1998, three rotations in 1997 and 1999 , and one rotation in 2000 .

Figure 2 shows examples of the flows at a depth of $7.1 \mathrm{Mm}$ determined over the full dense-pack mosaic on two adjacent days (3 and 4 April 1999). Although the flows are seen to evolve from day to day, they also possess more slowly evolving mean flow components. These are revealed by averaging many densepack maps over time and longitude: a weighted average of both the zonal and meridional flows is taken for all tiles of the dense-pack at a given latitude and depth for all the days in the period being analyzed for each year. 

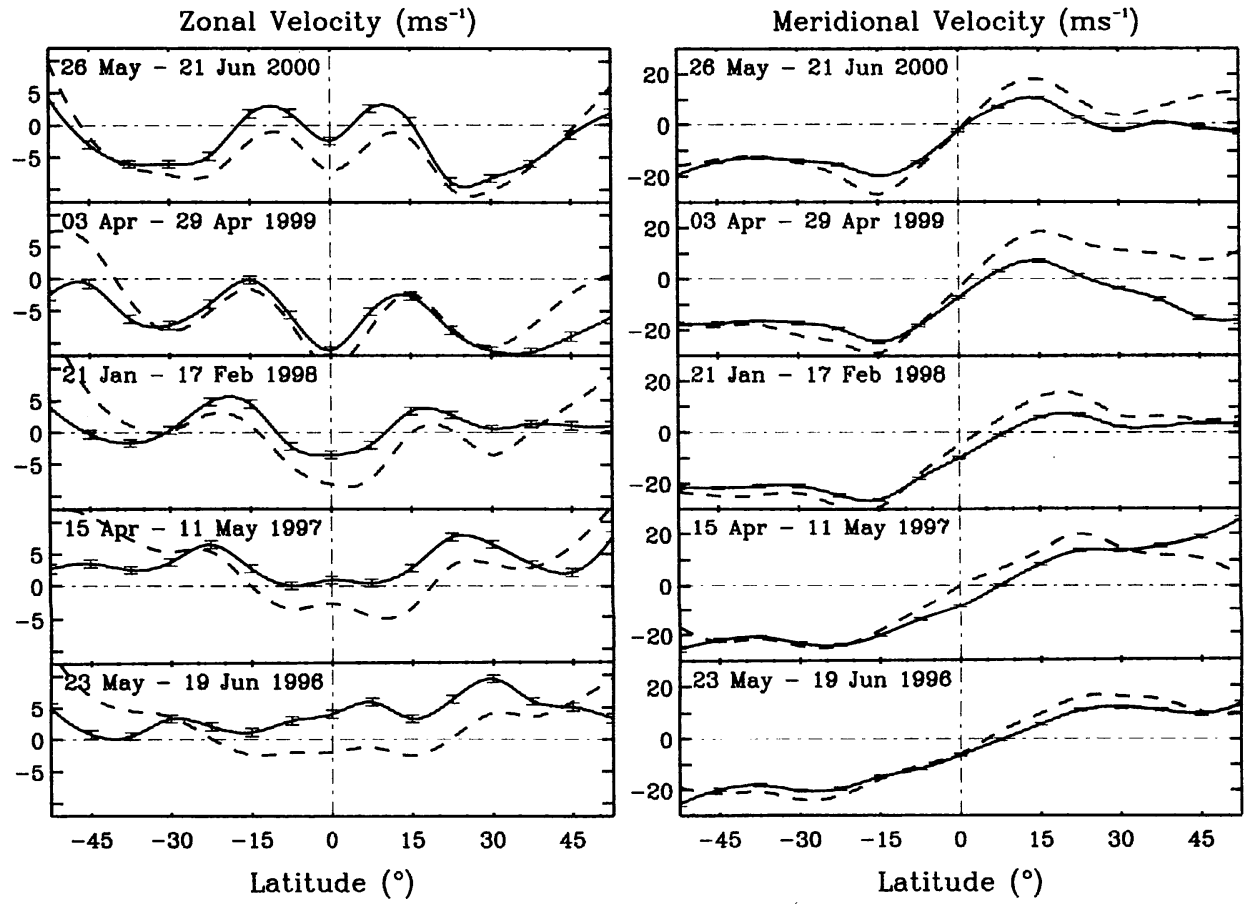

Figure 3. Mean zonal and meridional flows determined from longitudinal and temporal averages over the dense-pack mosaic for 25 consecutive days from each of the past 5 years. Shown are results of inversions at a depth of $1.2 \mathrm{Mm}$ (dashed curve) and at a depth of 7.1 Mm (solid curve).

\section{Evolving Mean Zonal and Meridional Flows}

Figure 3 shows the averaged zonal and meridional flows at depths of $1.2 \mathrm{Mm}$ and 7.1 $\mathrm{Mm}$ as determined from one full rotation period from each of the past five Dynamics Programs. To facilitate comparison, the zonal velocities at each depth have had their latitude and time means subtracted. Extending the averaging over more than one rotation does not significantly alter the overall results.

As noted in Haber et al. (2000), the longitudinally-averaged zonal velocity, after removing a smooth differential rotation component, possesses bands of fast and slow flow, much like the 'torsional oscillations' first reported from surface Doppler measurements (Howard and LaBonte, 1980) and recently from global helioseismic assessments (Schou, 1998). As the solar cycle progresses, the latitudes at which these fast bands occur migrate towards the equator. Our local-area analyses reveal that these belts of fast and slow flow are not symmetric about the solar equator, and the asymmetry changes with time. The asymmetry is most noticeable in 1996 for which the southern fast belt is nearly missing.

The meridional flows shown in Figure 3 are on the order of $20 \mathrm{~m} \mathrm{~s}^{-1}$ and are poleward for all years. These flows achieve maxima at the latitudes at which the zonal fast belts occur. As the zonal fast belts drift towards the equator, so do the latitudes of maximal meridional flow. The only exception to the poleward 
meridional flow occurs in 1999 when a two cell structure appears in the northern hemisphere. This high latitude cell is evident in the deeper inversions, such as $7.1 \mathrm{Mm}$, but is absent in the near surface layers. Except for this double cell structure, the meridional flow is roughly constant with depth from $2 \mathrm{Mm}$ down to $14 \mathrm{Mm}$ from the solar surface. The boundary between the low latitude and high latitude cell in 1999 occurs around latitude $20^{\circ}$ north for a depth of $7.1 \mathrm{Mm}$. In 1998 and in 2000 the meridional flow appears to be in transition between a single and double cell structure, whereas in 1996 and 1997 only a single cell was evident in both hemispheres.

Although the meridional flow is primarily poleward during most years, the latitude for which the meridional velocity vanishes does not necessarily occur at the equator. This asymmetry may be real but it is quite possible that measurement errors in the orientation angles of the sun have mixed some portion of the large rotational velocity into the meridional direction. Such errors could account for a meridional flow of up to about $10 \mathrm{~m} \mathrm{~s}^{-1}$. The meridional flow is not symmetric about the equator. However in the years 1996 to 1998 , the apparent asymmetry nearly vanishes if the curves are shifted such that the equator and the zero-point coincide. This is not the case in 1999 and 2000 where the asymmetry in meridional flow amplitude persists between the northern and southern hemispheres.

The existence of multicell structures for meridional flows within a single hemisphere has also been observed using time-distance helioseismology. Giles (1999) observed a second cell in the meridional flow in 1998 which appeared in the northern hemisphere at high latitudes, greater than $50^{\circ}$ north. The ringdiagram analyses for 1998 show a hint of such a flow below a depth of about 10 $\mathrm{Mm}$ from $45^{\circ}$ to $52.5^{\circ}$, the highest latitudes that the dense-pack studies reach. Thus the ring-diagram and time-distance results are quite similar. Typical flows within the near surface layers are $20 \mathrm{~m} \mathrm{~s}^{-1}$. Flows increase away from the equator, saturating at around $20^{\circ}$ north and south. A consistent interpretation of these properties is that a high latitude cell either drifts or expands southward between 1998 and 1999.

The asymmetries in the mean zonal flows and the detection of meridional flows below the surface are not feasible with global helioseismic techniques. The ability of ring-diagram analyses to measure these properties, and the variation of subsurface horizontal flows with position on the solar disk, makes ring-diagram analysis an important tool for studying the dynamics of the upper convection zone.

Acknowledgments. This research was supported by NASA through grants NAG 5-8133 and NAG 5-7996 and by NSF through grant ATM-9731676. The SOI-MDI project is supported by NASA grant NAG 5-3077 to Stanford University. SOHO is a project of international cooperation between ESA and NASA.

\section{References}

Giles, P. 1999, Ph.D. Thesis, Stanford University

Haber, D. A. et al. 2000, Solar Phys., 192, 335

Howard, R. and LaBonte, B. J. 1980, ApJ, 239, L33

Schou, J. et al. 1998, ApJ, 505, 390 